\title{
MACROSCOPIC LIMITS OF THE BECKER-DÖRING EQUATIONS
}

\author{
BARBARA NIETHAMMER *
}

Abstract. We review the derivation of macroscopic limits of the Becker-Döring equations. We show that those limits have the structure of a gradient flow even though the Becker-Döring equations themselves do not allow for such an interpretation.

\section{The Becker-Döring equations}

The special case of coagulation-fragmentation equations where clusters can gain or loose only one particle at a time are known as the Becker-Döring equations. They were originally developed [3] to describe nucleation of liquid droplets in a supersaturated vapor.

In the following we consider a homogeneous distribution of clusters which are characterized by their size $l$, the number of atoms in the cluster. We denote by $c_{l}(t)$ the concentration of $l$-clusters at time $t$.

The net rate of conversion of $l$-clusters into $(l+1)$-clusters is denoted by $J_{l}$, which is measured in units of clusters per unit time per unit volume. The rate of change of the density of $l$-clusters is thus given by

$$
\frac{d}{d t} c_{l}(t)=J_{l-1}(t)-J_{l}(t) \quad \text { for } l \geq 2 .
$$

The density of free atoms is then determined by the constraint that the total number of atoms is conserved, i.e.

$$
\rho:=\sum_{l=1}^{\infty} l c_{l}(t) \equiv \text { const. } \quad \text { for all } t \geq 0 .
$$

This implies with (1.1) that

$$
\frac{d}{d t} c_{1}(t)=-J_{1}-\sum_{l=1}^{\infty} J_{l} .
$$

To complete the system of equations we need a constitutive relation which gives $J_{l}$ in terms of $c_{l}$. As in chemical kinetics we assume

$$
J_{l}(t)=a_{l} c_{1}(t) c_{l}(t)-b_{l+1} c_{l+1}(t)
$$

with positive kinetic coefficients $a_{l}, b_{l}$ which are assumed to be independent of time. We assume in the following that the coefficients $a_{l}, b_{l}$ are given by

$$
a_{l}=l^{\alpha}, \quad b_{l}=a_{l}\left(z_{s}+\frac{q}{l \gamma}\right)
$$

where $\alpha \in[0,1), \gamma \in(0,1)$ and $z_{s}, q>0$ are parameters. These coefficients typically arise in density-conserving phase transitions such as the formation of liquid droplets in a supersaturated vapor or the phase segregation in a binary alloy after quenching. The Becker-Döring equations apply to the case of a non-uniform mixture, i.e. when

* Humboldt Universität zu Berlin, Institut für Mathematik, Unter den Linden 6, 10099 Berlin, Germany (niethamm@math.hu-berlin.de). 
the saturation density is small or respectively when one component of the alloy has small volume fraction. We refer to the appendix of [9] for a heuristic derivation of the coefficients (1.5) in several physically relevant cases. In particular we mention, that if clusters are spherical and transport of matter is dominated by diffusion of monomers, we obtain in three space dimensions $\alpha=\gamma=1 / 3$. In this application $z_{s}$ is just the equilibrium monomer concentration at a flat phase interface and $q$ is proportional to surface tension.

Existence, uniqueness and convergence to equilibrium has been established in the seminal mathematical paper [2] under quite general assumptions on the coefficients. Some refinements are contained in $[13,7]$.

By (1.1) equilibrium solutions $\left(\bar{c}_{l}\right)_{l}$ are given by $J_{l} \equiv$ const. for all $l$, but then due to (1.3) it must hold

$$
J_{l}=0 \quad \text { for } l \geq 1 \text {. }
$$

This implies

$$
\bar{c}_{l}=Q_{l} z^{l}, \quad l \geq 1
$$

with a parameter $z>0$, where $Q_{l}$ are given by

$$
Q_{1}=1, \quad \frac{Q_{l+1}}{Q_{l}}=\frac{a_{l}}{b_{l+1}} \quad \text { and thus } \quad Q_{l}=\frac{a_{1} a_{2} \cdots a_{l-1}}{b_{2} b_{3} \cdots b_{l}}
$$

Depending on the coefficients, the equilibrium density $\sum_{l=1}^{\infty} l Q_{l} z^{l}$ is bounded for $z$ in a certain range.

With the assumptions (1.5) we easily obtain for large $l$ that

$$
Q_{l} \cong \frac{C_{0}}{l^{\alpha} z_{s}^{l-1}} \exp \left\{-\frac{q}{(1-\gamma) z_{s}} l^{1-\gamma}\left(1+O\left(l^{-\gamma}\right)\right)\right\}
$$

Then the series $\sum_{l=1}^{\infty} l Q_{l} z^{l}$ has the convergence radius $\lim _{l \rightarrow \infty} \frac{b_{l+1}}{a_{l}}=z_{s}$ and the series also converges for $z=z_{s}$. In the following we denote

$$
\rho_{s}:=\sum_{l=1}^{\infty} l Q_{l} z_{s}^{l}<\infty
$$

which can be interpreted as the density of saturated vapor, and we use the notation $c_{l}^{s}=Q_{l} z_{s}^{l}, l=1,2, \ldots$ for the equilibrium configuration with density $\rho_{s}$.

Convergence of solutions to equilibrium was shown in [2] under some assumptions on coefficients and data, and was further generalized in $[1,13]$. The proof is based on the fact that there is a Lyapunov functional available, the free energy density, given by

$$
F(c)=\sum_{l=1}^{\infty} c_{l}\left(\ln \left(\frac{c_{l}}{Q_{l} z_{s}^{l}}\right)-1\right)+Q_{l} z_{s}^{l}
$$

In fact, it holds

$$
\frac{d}{d t} F(c(t))=-\sum_{l=1}^{\infty} J_{l} \ln \left(\frac{a_{l} c_{1} c_{l}}{b_{l+1} c_{l+1}}\right) \leq 0 .
$$


Since $F \geq 0$ it follows that $J_{l} \rightarrow 0$ as $t \rightarrow \infty$ such that $c_{l} \rightarrow Q_{l} z^{l}$ for some $z>0$. The question remains, what is $z$ and what happens to density conservation (1.2) in the limit as $t \rightarrow \infty$. It is shown in $[2,1,13]$ that if $\rho \leq \rho_{s}$ then $\lim _{t \rightarrow \infty} \sum_{l=1}^{\infty} l\left|c_{l}(t)-Q_{l} z^{l}\right|=$ 0 ,where $\rho=\sum_{l=1}^{\infty} l Q_{l} z^{l}$. However, if $\rho>\rho_{s}$, we have $\lim _{t \rightarrow \infty} c_{l}(t)=Q_{l} z_{s}^{l}$ for each $l \geq 1$, but the convergence is only weak and the density drops to $\rho_{s}$ in the limit $t \rightarrow \infty$. The so called excess density is contained in larger and larger clusters as time evolves. In phase transformations these large clusters represent the stable nuclei of the new thermodynamic phase, e.g. the liquid droplets formed out of the supersaturated vapor. This can however happen very slowly. In fact, existence of metastable states in the Becker-Döring model has been established in [11]. More precisely, specific solutions of the Becker-Döring equations are constructed, for data with density $\rho>\rho_{s}$, which stay very long, that is at least exponentially long in $1 /\left(\rho-\rho_{s}\right)$, close to the data, before they converge to their corresponding equilibrium.

\section{Dynamics of large clusters: heuristics}

We now investigate the governing dynamics of the large clusters which form the new thermodynamic phase, once any possible metastable state has broken down.

We recall briefly an argument, which has been given in [12] for the case $\alpha=\gamma=$ $1 / 3$, for the general case when coefficients are given by (1.5). To consider large times a new time scale $\tau=\varepsilon^{1+\gamma-\alpha} t$ is introduced for a small parameter $\varepsilon \rightarrow 0$, such that

$$
\frac{d}{d \tau} c_{l}=\frac{1}{\varepsilon^{1+\gamma-\alpha}}\left(J_{l-1}-J_{l}\right)
$$

We write

$$
J_{l}=a_{l}\left(\left(c_{1}-z_{s}\right)-q\right) c_{l}-\left(b_{l+1} c_{l+1}-b_{l} c_{l}\right)
$$

and choose as a cut between small and large clusters $l_{0}=l_{0}(\varepsilon)$ which can be chosen as $l_{0}=\ln \left(\frac{1}{\varepsilon}\right)$ for example. For $l \geq l_{0}$ one substitutes $\lambda=\varepsilon l$ and treats $\lambda$ as a continuous variable. Furthermore one introduces the rescaled cluster densities and fluxes as

$$
\begin{aligned}
c_{l} & =\varepsilon^{2} \nu(\lambda, \tau), \\
J_{l} & =\varepsilon^{2-\gamma+\alpha} v(\lambda, \tau),
\end{aligned}
$$

and the rescaled monomer density

$$
c_{1}-z_{s}=\varepsilon^{\gamma} u(\tau)
$$

Plugging this ansatz into (2.1) and (2.2) gives to leading order

$$
\partial_{\tau} \nu+\partial_{\lambda}\left(\lambda^{\alpha}\left(u-\frac{q}{\lambda^{\gamma}}\right) \nu\right)=0 .
$$

For the small clusters one can argue as in [12] that for large times the cluster densities are already close to equilibrium such that

$$
c_{l}=Q_{l} z_{s}^{l}(1+o(1))
$$


Density conservation (1.2) now gives

$$
\begin{aligned}
\rho=\sum_{l=1}^{\infty} l c_{l} & =\sum_{l=1}^{l_{0}-1} l c_{l}+\sum_{l=l_{0}}^{\infty} l c_{l} \\
& =\sum_{l=1}^{l_{0}-1} l Q_{l} z_{s}^{l}+\sum_{l=l_{0}}^{\infty} l c_{l}+o(1) \\
& =\rho_{s}+\int \lambda \nu(\lambda, \tau) d \lambda+o(1) .
\end{aligned}
$$

We obtain to leading order

$$
\int \lambda \nu d \lambda=\rho-\rho_{s}
$$

which together with $(2.6)$ is equivalent to

$$
u(\tau)=\frac{q \int \lambda^{\alpha-\gamma} \nu d \lambda}{\int \lambda^{\alpha} \nu d \lambda}
$$

For the case $\alpha=\gamma=1 / 3$ system (2.6), (2.9) is just the classical LSW model for coarsening (cf. [8, 14]), which describes the large time evolution of the stable clusters of the new thermodynamic phase. Once, the new phase has emerged, the free energy is dominated by surface energy attached to the interface between clusters and the ambient phase. Thus, the system is driven by the reduction of this surface energy and limited by diffusion of atoms between the clusters. As a consequence, large particle grow and smaller ones shrink, such that the typical cluster sizes increases whereas the number decreases.

\section{Rigorous derivation of large cluster dynamics}

The arguments of [12], have been justified rigorously in [9] for the class of coefficients given by (1.5). The key idea in the analysis is to exploit the energy estimate implied by (1.10). For that it is instructive to write $F$ in the following way:

$$
F(c(t))=\sum_{l=1}^{\infty} c_{l}\left(\ln \left(\frac{c_{l}}{c_{l}^{s}}\right)-1\right)+c_{l}^{s}=\sum_{l=1}^{\infty} c_{l}^{s} f\left(\frac{c_{l}-c_{l}^{s}}{c_{l}^{s}}\right)
$$

with $f(z)=(1+z) \ln (1+z)-z$, which resembles the notion often used in the study of the Boltzmann equation, where $F$ is usually called the relative entropy. We also notice that $f$ behaves quadratically for bounded $\frac{c_{l}-c_{l}^{s}}{c_{l}^{s}}$, whereas the growth is only superlinear for large $\frac{c_{l}-c_{l}^{s}}{c_{l}^{s}}$, which is the case for the large clusters.

We now ask for the leading order term in the energy for large clusters. Using (1.7) we find

$$
\begin{aligned}
\sum_{l=l_{0}}^{\infty} c_{l}\left(\ln \left(\frac{c_{l}}{c_{l}^{s}}\right)-1\right)+c_{l}^{s} & =\sum_{l=l_{0}}^{\infty} c_{l} \ln \left(\frac{1}{Q_{l} z_{s}^{l}}\right)+\sum_{l=l_{0}}^{\infty} c_{l}\left(\ln c_{l}-1\right)+c_{l}^{s} \\
\approx & \frac{q}{z_{s}(1-\gamma)} \sum_{l=l_{0}}^{\infty} l^{1-\gamma} c_{l}+O\left(\sum_{l=l_{0}}^{\infty} l^{1-2 \gamma} c_{l}\right) \\
& +\sum_{l=l_{0}}^{\infty} c_{l}\left(\ln c_{l}-1\right)+c_{l}^{s}
\end{aligned}
$$


It is easily seen that $\sum_{l=l_{0}}^{\infty} c_{l}\left(\ln c_{l}-1\right)=o\left(\sum_{l=l_{0}}^{\infty} l^{1-\gamma} c_{l}\right)$ and the same holds for $\sum_{l=l_{0}}^{\infty} c_{l}^{s}$ if $l_{0}$ is just moderately large, since $\left(c_{l}^{s}\right)$ is decreasing exponentially fast. Thus, the leading order term in the energy is $\frac{q}{z_{s}(1-\gamma)} \sum_{l=l_{0}}^{\infty} l^{1-\gamma} c_{l}$, which in typical applications is just the surface energy density of the clusters. Recalling the scaling introduced in the beginning of Section 2 we find

$$
\frac{q}{z_{s}(1-\gamma)} \sum_{l=l_{0}}^{\infty} l^{1-\gamma} c_{l} \approx \varepsilon^{\gamma} \frac{q}{z_{s}(1-\gamma)} \int \lambda^{1-\gamma} \nu d \lambda
$$

This indicates that a natural criterion for the system being in the last stage is, that the energy scales like $\varepsilon^{\gamma}$ if $\varepsilon^{-1}$ is a measure for the large clusters. Correspondingly we will assume that we have a sequence of data such that

$$
F\left(c^{\varepsilon}(0)\right)=\varepsilon^{\gamma} .
$$

We introduce a new time scale and rescaled monomer density as in Section 2. The cut-off $l_{0}=l_{0}(\varepsilon)$ between small and large clusters needs to satisfy

$$
\begin{aligned}
\left|c_{l_{0}}^{s}\right|^{\eta} & \leq C F\left(c^{\varepsilon}(0)\right) \quad \text { for any } \eta>0, \\
\lim _{\varepsilon \rightarrow 0} l_{0}^{\gamma} \sqrt{F\left(c^{\varepsilon}(0)\right)} & =0 .
\end{aligned}
$$

These requirements are needed to ensure, that on the one hand $l_{0}$ is large enough such that some moment of the rescaled size distribution is bounded and on the other hand, $l_{0}$ is small enough such that the excess density is contained in the clusters larger than $l_{0}$.

The rescaled densities are defined as the properly rescaled empirical measures $\left\{\nu_{t}^{\varepsilon}\right\}_{t} \subset C_{0}^{0}\left(\mathbb{R}^{+}\right)^{*}$ via

$$
\int_{0}^{\infty} \zeta(\lambda) d \nu_{\tau}^{\varepsilon}:=\frac{1}{\varepsilon} \sum_{l=l_{0}}^{\infty} \zeta(\varepsilon l) c_{l}^{\varepsilon}(t)
$$

For the rigorous derivation of (2.6), (2.8) one has to make one assumption on the data, which is that at the time when the energy is appropriately small as in (3.2), not too many very large clusters have formed. More precisely we assume

$$
\sum_{l \geq[M / \varepsilon]}^{\infty} l c_{l}^{\varepsilon}(0)=\int_{\lambda \geq M} \lambda d \nu_{0}^{\varepsilon} \rightarrow 0 \quad \text { as } M \rightarrow \infty \text { uniformly in } \varepsilon .
$$

Theorem 3.1. ([9], Theorem 1.1) Assume that (3.2) and (3.4) hold. For a subsequence, again denoted by $\varepsilon \rightarrow 0$, it holds $\nu_{\tau}^{\varepsilon} \rightarrow \nu_{\tau}$ locally uniformly in time in the sense of measures and $u^{\varepsilon} \rightarrow u$ in $L_{l o c}^{2}([0, \infty))$. The limit satisfies

$$
\begin{aligned}
\partial_{\tau} \nu_{\tau}+\partial_{\lambda}\left(\lambda^{\alpha}\left(u(\tau)-\frac{q}{\lambda^{\gamma}}\right) \nu_{\tau}\right) & =0, & \text { in } \mathcal{D}^{\prime}, \\
\int \lambda d \nu_{\tau} & =\rho-\rho_{s} \quad & \text { for all } \tau \geq 0 .
\end{aligned}
$$

The key ingredient in the proof of Theorem 3.1 is the following estimate, which for the sake of lucidity we state separately. 
Proposition 3.1. ([9], Proposition 1.2) The solution $\nu_{t}$, found in Theorem 3.1, satisfies the following energy estimate: For any $\tau>0$ we have

$$
\begin{aligned}
\frac{q}{z_{s}(1-\gamma)} \int \lambda^{1-\gamma} d \nu_{\tau}+\frac{1}{z_{s}} \int_{0}^{\tau} \int \lambda^{\alpha}(u(s)- & \left.\frac{q}{\lambda^{\gamma}}\right)^{2} d \nu_{s} d s \\
& \leq \liminf _{\varepsilon \rightarrow 0} \frac{F\left(c^{\varepsilon}(0)\right)}{\varepsilon^{\gamma}} \leq 1 .
\end{aligned}
$$

The detailed proofs of Theorem 3.1 and Proposition 3.1 are contained in Section 2 of [9]. In fact, the energy estimate implied by (1.10) yields control on both, the surface energy of the large clusters, as well as the deviation of small clusters from equilibrium. This implies that the excess density is contained in the large clusters. The control on the dissipation rate of the energy implies that assumption (3.4) is preserved in time. Those estimates imply weak compactness of $\nu_{\tau}^{\varepsilon}$. It remains to identify the limit flux. The main problem here is that there is a lack of a-priori estimates on $u^{\varepsilon}$. Nevertheless, an indirect argument, using again the control on the dissipation rate of the energy, gives weak compactness of $u^{\varepsilon}$.

The main question which remains open is, whether assumption (3.4) is a natural one. More precisely, one considers the evolution of clusters under the Becker-Döring equations for generic data, e.g. only monomers. Then, at the time when the energy is appropriately small according to (3.2) one has to check whether assumption (3.4) is satisfied. Numerical simulations and heuristic arguments give no indication that very large clusters are created such that (3.4) is violated. A rigorous proof is however not available yet and seems difficult to obtain due to the possible appearance of metastable states.

A first attempt to understand in a rigorous manner the return to equilibrium in the Becker-Döring model has so far only been undertaken in the case of subcritical density in [6]. There, exponentially fast convergence to equilibrium has been shown also using the energy-energy-dissipation relation.

In [7] one can find another rigorous derivation of (2.6) and (2.9), complementary to ours, for homogeneous coefficients, i.e. (1.5) with the assumption $z_{s}=0$ and $\alpha \geq \gamma$. In this case there exists no nontrivial equilibrium and no useful Lyapunov functional; but one can in this special case use (1.3) to derive a uniform bound on $u^{\varepsilon}$.

Finally, let us mention a modification to (2.6), (2.9), which is often considered in the literature, where mass in the diffusion field $u$ is taken into account, whence mass conservation takes the form

$$
A u(\tau)+\int \lambda d \nu_{\tau}=\text { const. } \quad \text { for all } \tau>0
$$

and some constant $A$. In fact, (2.6) together with (3.7) has been derived as a macroscopic limit of the Becker-Döring equations in [4], however for a different type of coefficients than considered in this note.

\section{Gradient flow structure of macroscopic limits}

In this section we briefly describe the gradient flow structure of the macroscopic limits (2.6) coupled with either (2.8) or (3.7). An immediate consequence of this structure is the energy estimate (3.6) on which the proof of Theorem 3.1 is based and which has also been exploited in [5] and [10]. However, it is worth noticing that the Becker-Döring equations do not allow for an interpretation as a gradient flow. 
The concept of an abstract gradient flow consists of a differentiable manifold $\mathcal{M}$, a metric tensor $g$ on $\mathcal{M}$ and an energy functional $E$. The dynamical system $\dot{x}=-\operatorname{grad} E(x)$ defines the gradient flow of $E$ on $(\mathcal{M}, g)$ and as it's most important feature the energy decreases along trajectories.

We first describe the gradient flow structure of (2.6), (2.8). This evolution preserves the first moment and thus the manifold is given by

$$
\mathcal{M}:=\left\{\nu: \mathbb{R}_{+} \rightarrow \mathbb{R}_{+} \mid \int \lambda \nu d \lambda=\rho_{0}\right\}
$$

The tangent space is correspondingly given by functions with vanishing first moment:

$$
T_{\nu} \mathcal{M}:=\left\{s: \mathbb{R}_{+} \rightarrow \mathbb{R} \mid \int \lambda s d \lambda=0\right\} .
$$

To define the metric tensor $g$ we identify the tangent space with

$$
T_{\nu} \mathcal{M} \cong\left\{v: \mathbb{R}_{+} \rightarrow \mathbb{R} \mid \int v \nu d \lambda=0\right\}
$$

where the identification is defined via $s+\partial_{\lambda}(v \nu)=0$. Then the metric tensor is given by

$$
g_{\nu}\left(s_{1}, s_{2}\right)=\int \frac{v_{1} v_{2}}{\lambda^{\alpha}} \nu d \lambda
$$

The energy of the system is

$$
E(\nu)=\frac{q}{z_{s}(1-\gamma)} \int \lambda^{1-\gamma} \nu d \lambda
$$

and its differential via

$$
D E(\nu) s=\frac{q}{z_{s}(1-\gamma)} \int \lambda^{1-\gamma} s d \lambda=\frac{q}{z_{s}} \int \lambda^{-\gamma} v \nu d \lambda .
$$

The gradient flow is now for all $\tau>0$ defined via

$$
g_{\nu_{\tau}}\left(\partial_{\tau} \nu, s\right)+D E(\nu) s=0 \quad \text { for all } s \in T_{\nu} \mathcal{M}
$$

In view of (4.1) and (4.3) equation (4.4) is equivalent to

$$
\int \frac{v \tilde{v}}{\lambda^{\alpha}} \nu d \lambda=-\frac{q}{z_{s}} \int \lambda^{-\gamma} \tilde{v} \nu d \lambda \quad \text { for all } \tilde{v} \in T_{\nu} \mathcal{M}
$$

where

$$
\partial_{\tau} \nu=-\partial_{\lambda}(v \nu)
$$

The relation (4.5) implies

$$
v \lambda^{-\alpha}=u(\tau)-\frac{q}{z_{s}} \lambda^{-\gamma},
$$

where $u(\tau)$ is a Lagrange multiplier determined by the constraint $\int v \nu=0$. Thus, (4.6) and (4.7) are just (2.6). 
Using the above concepts it is easily seen that (2.6), (3.7) just corresponds to the unconstrained gradient flow with respect to the penalized energy

$$
E(\nu)=\frac{q}{z_{s}(1-\gamma)} \int \lambda^{1-\gamma} \nu d \lambda+\frac{u^{2}}{2 A}
$$

with $u=\rho_{0}-\int \lambda \nu d \lambda$.

\section{REFERENCES}

[1] J. Ball and J. Carr, Asymptotic behavior of solutions to the Becker-Döring equations for arbitrary initial data, Proc. R. Soc. Edinb., Sect. A, 108(1/2):109-116, 1988.

[2] J. Ball, J. Carr, and O. Penrose, The Becker-Döring cluster equations: basic properties and asymptotic behaviour of solutions, Comm. Math. Phys., 104:657-692, 1986.

[3] R. Becker and W. Döring, Kinetische Behandlung der Keimbildung in übersättigten Dämpfen, Ann. Phys., (Leipzig), 4:719-752, 1935.

[4] J.F. Collet, T. Goudon, F. Poupaud, and A. Vasseur, The Becker-Döring system and its Lifshitz-Slyozov limit, SIAM J. Appl. Math, 62(5):1488-1500, 2002.

[5] J.F. Collet, T. Goudon, and A. Vasseur, Some remarks on large-time asymptotics of the Lifshitz-Slyozov equations, J. Stat. Phys., 108(1-2):341-359, 2002.

[6] P.E. Jabin and B. Niethammer, On the rate of convergence to equilibrium in the Becker-Döring equation, J. Differential Equations, 191(2):518-543, 2003.

[7] P. Laurençot and S. Mischler, From the Becker-Döring to the Lifshitz-Slyozov-Wagner equations, J. Stat. Phys., 106(5-6):957-991, 2002.

[8] I.M. Lifshitz and V.V. Slyozov, The kinetics of precipitation from supersaturated solid solutions, J. Phys. Chem. Solids, 19:35-50, 1961.

[9] B. Niethammer, On the evolution of large clusters in the Becker-Döring model, J. Nonlinear Science, 31(1):115-155, 2003.

[10] B. Niethammer and R. L. Pego, Well-posedness for measure transport in a family of nonlocal domain coarsening models, to appear in Indiana Univ. Math. journal, 2004 .

[11] O. Penrose, Metastable states for the Becker-Döring cluster equations, Comm. Math. Phys., 124:515-541, 1989.

[12] O. Penrose, The Becker-Döring equations at large times and their connection with the LSW theory of coarsening, J. Stat. Phys., 89(1/2):305-320, 1997.

[13] M. Slemrod, Trend to equilibrium in the Becker-Döring cluster equations, Nonlinearity, 2(3):429-443, 1989.

[14] C. Wagner, Theorie der alterung von niederschlägen durch umlösen, Z. Elektrochemie, 65:581594, 1961. 